\title{
Exosomal ANGPTL1 attenuates colorectal cancer liver metastasis by regulating Kupffer cell secretion pattern and impeding MMP9 induced vascular leakiness
}

Kai Jiang ${ }^{1,2+}$, Haiyan Chen ${ }^{1,2 \dagger}$, Yimin Fang ${ }^{1,2 \dagger}$, Liubo Chen ${ }^{1,2}$, Chenhan Zhong ${ }^{2,3}$, Tongtong Bu ${ }^{1}$, Siqi Dai ${ }^{1,2}$, Xiang Pan ${ }^{1,2}$, Dongliang Fu ${ }^{1,2}$, Yucheng Qian ${ }^{1,2}$, Jingsun Wei ${ }^{1,2}$ and Kefeng Ding ${ }^{1,2^{*}}$

\begin{abstract}
Background: Angiopoietin-like protein 1 (ANGPTL1) has been proved to suppress tumor metastasis in several cancers. However, its extracellular effects on the pre-metastatic niches (PMNs) are still unclear. ANGPTL1 has been identified in exosomes, while its function remains unknown. This study was designed to explore the role of exosomal ANGPTL1 on liver metastasis in colorectal cancer (CRC).

Methods: Exosomes were isolated by ultracentrifugation. The ANGPTL1 level was detected in exosomes derived from human CRC tissues. The effects of exosomal ANGPTL1 on CRC liver metastasis were explored by the intrasplenic injection mouse model. The liver PMN was examined by vascular permeability assays. Exosomal ANGPTL1 localization was validated by exosome labeling. The regulatory mechanisms of exosomal ANGPTL1 on Kupffer cells were determined by RNA sequencing. QRT-PCR, Western Blot, and ELISA analysis were conducted to examine gene expressions at mRNA and protein levels.

Results: ANGPTL1 protein level was significantly downregulated in the exosomes derived from CRC tumors compared with paired normal tissues. Besides, exosomal ANGPTL1 attenuated liver metastasis and impeded vascular leakiness in the liver PMN. Moreover, exosomal ANGPTL1 was mainly taken up by KCs and regulated the KCs secretion pattern, enormously decreasing the MMP9 expression, which finally prevented the liver vascular leakiness. In mechanism, exosomal ANGPTL1 downregulated MMP9 level in KCs by inhibiting the JAK2-STAT3 signaling pathway.

Conclusions: Taken together, exosomal ANGPTL1 attenuated CRC liver metastasis and impeded vascular leakiness in the liver PMN by reprogramming the Kupffer cell and decreasing the MMP9 expression. This study suggests a suppression role of exosomal ANGPTL1 on CRC liver metastasis and expands the approach of ANGPTL1 functioning.
\end{abstract}

Keywords: Exosomal ANGPTL1, Colorectal Cancer, Liver metastasis, PMNs, Kupffer cell, MMP9

\footnotetext{
* Correspondence: dingkefeng@zju.edu.cn

${ }^{\dagger}$ Kai Jiang, Haiyan Chen and Yimin Fang are co-first authors.

'Department of Colorectal Surgery and Oncology, Key Laboratory of Cancer Prevention and Intervention, Ministry of Education, the Second Affiliated Hospital, Zhejiang University School of Medicine, Hangzhou, Zhejiang, China ${ }^{2}$ Cancer Institute, Key Laboratory of Cancer Prevention and Intervention, Ministry of Education, The Second Affiliated Hospital, Zhejiang University School of Medicine, Hangzhou, Zhejiang, China

Full list of author information is available at the end of the article
} changes were made. The images or other third party material in this article are included in the article's Creative Commons licence, unless indicated otherwise in a credit line to the material. If material is not included in the article's Creative Commons licence and your intended use is not permitted by statutory regulation or exceeds the permitted use, you will need to obtain permission directly from the copyright holder. To view a copy of this licence, visit http://creativecommons.org/licenses/by/4.0/ The Creative Commons Public Domain Dedication waiver (http://creativecommons.org/publicdomain/zero/1.0/) applies to the data made available in this article, unless otherwise stated in a credit line to the data. 


\section{Background}

Colorectal cancer (CRC) is one of the most common digestive tract malignancies worldwide with high prevalence and mortality [1]. Distant metastasis is the leading cause of cancer-associated death in CRC patients [2]. The 5-year survival rate is $92 \%$ for patients with local disease, while it sharply declines to 53 and $11 \%$ for patients with regional and distant metastasis [3]. The liver is the most common site of CRC metastasis. It is reported that $\leq 25 \%$ of CRC patients have synchronous colorectal liver metastases (CLM) upon diagnosis, and up to half of CRCs will eventually lead to liver metastasis [4]. Despite advances in surgical technique and targeted therapy, CLM patients' prognosis is still poor [5]. It is urgent to explore the mechanism of liver metastasis and seek a new strategy for CLM treatment.

In our previous study [6], we found some of the angiopoietin-like proteins (ANGPTLs) were downregulated in CRC tissue, among which ANGPTL1 was the most significant one. ANGPTLs are a family of proteins similar to angiopoietins in structure, including ANGPTL1 to ANGP TL8 [7]. They were reported to affect angiogenesis [7], inflammation [8], metabolism disorders [9], hematopoiesis [10], and cancer development [11, 12]. Early studies showed that Angiopoietin-like protein 1 (ANGPTL1) acts as an antiangiogenic factor and a tumor suppressor [13, 14]. ANGP TL1 is downregulated in various cancers $[6,7]$, and several studies have proved its inhibitory role in tumor growth and metastasis [15-18]. Our previous study also demonstrated that ANGPTL1 overexpression inhibited the migration and invasion of CRC cells, leading to liver metastasis suppression. Besides, low expression of ANGPTL1 was related to poorer prognosis in CRC patients [6].

Nevertheless, previous researches about ANGPTL1's function limited in primary tumors $[6,16,18]$. As a secretory protein [14], the biological effects of extracellular ANGPTL1 on the metastatic organs are still under investigation. Increasing evidence shows that the primary tumor-secreted factors and exosomes can enhance metastasis by promoting a supportive microenvironment in the metastatic organs, named the premetastatic niches (PMNs). The PMNs include several characteristics, such as vascular leakiness, inflammation, and immunosuppression [19]. Exosomes are small extracellular vesicles ranging from 50 to $160 \mathrm{~nm}$ in size carrying proteins, nucleic acids, and lipids [20]. Recently, tumor-derived exosomes have been reported to be involved in PMNs formation $[21,22]$. As the earliest event during PMNs evolution, vascular permeability is always regulated by tumor-derived exosomes [23]. For instance, CRC-derived exosomal miR-25-3p can promote PMNs formation by inducing vascular permeability [24]; breast cancer-derived exosomal miR-105 can destroy vascular endothelial barriers to promote metastasis [25]. Interestingly, ANGPTL1 has been identified in exosomes derived from saliva [26], urine [27], and ovarian cancer cells [28]. Nevertheless, the function of exosomal ANGPTL1 in CRC is still unknown.

In this study, we focused on the role of exosomal ANGPTL1 in CRC metastasis. We studied the protein level of ANGPTL1 in exosomes derived from tumor and normal tissues in CRC patients. Both In vivo and in vitro models were applied to characterize the effects of exosomal ANGPTL1 on CRC liver metastasis and PMNs formation. We demonstrated that exosomal ANGPTL1 attenuates CRC liver metastasis by regulating the kupffer cell secretion pattern and impeding vascular leakiness in the liver PMN.

\section{Methods}

\section{Patients and specimens}

The clinical CRC and paired normal tissues were obtained from CRC patients $(n=8)$ in the Second Affiliated Hospital of Zhejiang University School of Medicine. This project was approved by the ethical committee of the Second Affiliated Hospital of Zhejiang University School of Medicine. Informed consent was obtained from all patients.

\section{Cell culture}

The human colorectal cancer cell line SW620 and Human umbilical vein endothelial cell (HUVEC) were obtained from the American Type Culture Collection (Rockville, MD, USA). The ANGPTL1 stably overexpressed cell line (SW620-ANGPTL1) and its control cell line (SW620-Ctrl) were established in the previous study [6]. The Immortalized Mouse Kupffer Cell line (ImKC) was purchased from MilliporeSigma (USA). SW620 cells and HUVECs were cultured in RMPI-1640 medium (Gibco, Carlsbad, CA) supplemented with 10\% fetal bovine serum (FBS, Gibco, Brazil). ImKC were cultured in DMEM medium (Gibco, Carlsbad, CA) supplemented with 10\% FBS (Gibco, Brazil).

\section{Exosome collection and characterization}

Exosomes were collected by sequential ultracentrifugation. CRC cells were cultured in the exosome-depleted $(160,000 \times g, 16 \mathrm{~h})$ complete medium for $72 \mathrm{~h}$. The supernatants were collected and centrifugate at $500 \times \mathrm{g}$ for 10 min to remove cell contamination, then at $3000 \times \mathrm{g}$ for $20 \mathrm{~min}$ to remove apoptotic bodies and large cell debris, followed by centrifugation at $12,000 \times \mathrm{g}$ for $20 \mathrm{~min}$ to remove large microvesicles. Next, exosomes were collected by $100,000 \times$ g centrifugation for $70 \mathrm{~min}$ (Beckman Ti70). The exosome pellet was resuspended in $20 \mathrm{~mL}$ of phosphate-buffered saline (PBS) and collected by 100 , $000 \times$ g ultracentrifugation for $70 \mathrm{~min}$ (Beckman Ti70). Exosome preparation was verified by Transmission electron microscopy (TEM). Exosome size was measured by dynamic light scattering (DLS) analysis using Zetasizer Nano ZSE (Malvern Panalytical, Shanghai, China). 
For tissue-derived exosome collection, the CRC tumors and paired normal tissues were cut into $1 \mathrm{~mm} \times 1$ $\mathrm{mm}$ pieces and cultured in $15 \mathrm{~mL}$ of FBS-free RMPI1640 medium for $24 \mathrm{~h}$. Then the supernatant was harvested for further isolation of exosomes.

\section{Exosome treatment and labeling}

Purified exosomes were injected into the mouse retroorbital venous sinus in a total volume of $100 \mu \mathrm{L}$ PBS. For in vivo education experiments, mice received $5 \mu \mathrm{g}$ of exosomes every other day for 21 days. Retro-orbital injection of PBS was used in control groups. For in vitro education, exosomes $(10 \mu \mathrm{g} / \mathrm{mL})$ were added into the culture medium $(\mathrm{CM})$ of $\operatorname{ImKC}$ for $3,6,12,24 \mathrm{~h}$. For exosome-tracking experiments, exosomes were labeled using PKH67 membrane dye (Sigma, Shanghai, China), followed by $100,000 \times \mathrm{g}$ ultracentrifugation for $70 \mathrm{~min}$, and labeled exosomes were resuspended in PBS. In experiments involving the evaluation of exosome incorporation, labeled exosomes were injected retro-orbitally into the mice or added into the CM of ImKC $24 \mathrm{~h}$ before immunofluorescence analysis for exosome cells.

\section{Animal model}

To analyze the role of exosomal ANGPTL1 in CRC liver metastasis, we pre-educated the 6-8-week-old scid-beige mice (SLAC Laboratory Animal Co. Ltd., Shanghai, China) retro-orbitally with exosomes for 21 days $(5 \mu \mathrm{g} /$ 100ul, every other day). Then, $2 \times 10^{6} \mathrm{SW} 620$ cells were injected into the mice spleen as described in the previous study $[29,30]$. A small animal IVIS Lumina Imaging System (Caliper Life Sciences, Hopkinton, MA) was used for liver lesion monitor. All mice were sacrificed at one month, and the livers were harvested for Hematoxylin and eosin (H\&E) staining and analysis of metastases. For liver macrophage elimination, liposome clodronate was injected via the tail vein in a dose of $0.2 \mathrm{~mL} / 20-25 \mathrm{~g}$ as a tool to suppress macrophage function by inducing apoptosis [31]. Liposomes containing PBS were injected as a control. All animal experiments were approved by the Institutional Ethics Committee of the Second Affiliated Hospital Zhejiang University School of Medicine.

\section{In vivo vascular permeability assay}

After pre-education with exosomes for 21 days, mice were injected with the recombinant mouse MMP9 (rmMMP9; $50 \mu \mathrm{g} / \mathrm{kg}$ body weight; R\&D) intravenously. The rmMMP9 was preactivated using $1 \mathrm{mM}$ aminophenylmercuricacetate (AMPA, Sigma, Shanghai, China) for $2 \mathrm{~h}$ at $37^{\circ} \mathrm{C}$. One hour after rmMMP9 injection, FITCDextran ( 70KD; $100 \mathrm{mg} / \mathrm{kg}$; Sigma, Shanghai, China) was injected through the tail vein. After one hour, mice were sacrificed and perfused with PBS to remove the excess dye. Liver tissues were embedded in Tissue-Tek
O.C.T. Compound (Sakura; Torrance, CA, USA) to make frozen blocks for sectioning and immunofluorescent staining. Stained sections were viewed and photographed with a fluorescence microscope. The intensity of fluorescence was measured using ImageJ software (ImageJ software v1.8.0).

\section{Endothelial permeability}

HUVECs $\left(2 \times 10^{4}\right)$ were seeded on transwell filters $(0.4 \mu \mathrm{m}$ pore size; Corning, Shanghai, China). After reaching confluence, HUVECs were treated with CM from ImKC educated by PBS, Ctrl-Exo, or ANGPTL1Exo (with or without rmMMP9, $100 \mathrm{ng} / \mathrm{mL}$ ) for $48 \mathrm{~h}$. Then, FITC-Dextran $(1 \mathrm{mg} / \mathrm{mL})$ was added to the top well. $40 \mu \mathrm{L}$ medium in the bottom well was taken for fluorescence measurement every 30 min using a SpectraMax microplate reader (SpectraMax i3, Molecular Devices, USA) at $488 \mathrm{~nm}$ excitation and $520 \mathrm{~nm}$ emission. The fluorescence intensity represents the passage of FITC-Dextran, which represents the the HUVECs layer permeability.

\section{Small interfering (siRNA) transfection}

Mouse MMP9 siRNA was purchased from Tsingke biological technology (Hangzhou, China). The MMP9 siRNA and scramble siRNA were added into the ImKC $24 \mathrm{~h}$ after exosome pre-education. The siRNA transfection was performed with Lipofectamine 3000 (Invitrogen, Carlsbad, CA) according to the manufacturer's instructions. The siRNA sequences were as follows: MMP9 siRNA, 5'CAAGACAAAGCCUAUUUCUTT-3' (sense), 5' -AGAA AUAGGCUUUGUCUUGTT-3' (antisense).

\section{qRT-PCR}

Total RNAs were isolated from cells and mouse livers with TRIzol reagent (Invitrogen, USA) and evaluated using NanoDrop 2000 spectrophotometer (Thermo Scientific, Pittsburgh, PA, USA). qRT-PCR was conducted using a standard SYBR-Green PCR kit protocol (YEAS EN, Shanghai, China) with a 7500 Fast Real-Time PCR System (Life Technologies, Shanghai, China). The primers were synthesized by Tsingke biological technology (Hangzhou, China). The sequences of all primers are listed in Table 1.

\section{RNA sequencing}

Total RNA of ImKC cells educated by PBS or exosomes for $24 \mathrm{~h}$ in vitro was extracted and subjected to BGI (Huada Genomics Institute Co. Ltd., Guangzhou, China) for RNA sequencing. The DEGseq $\mathrm{R}$ package was used to analyze differentially expressed genes based on the conditions of a fold change $(\mathrm{FC}) \geq 1$ and Q-values $\leq 0.001$. Gene Ontology (GO) and Kyoto Encyclopedia of Genes and Genomes (KEGG) pathway enrichment 
Table 1 Primers used for quantitative real-time PCR were $\left(5^{\prime}-3^{\prime}\right)$

\begin{tabular}{lll}
\hline Genes & Forward & Reverse \\
\hline Gapdh & GGTGAAGGTCGGTGGAACG & CTCGCTCCTGGAAGATGGTG \\
II-1a & ATTGTGCCCTTACTGCTGCTG & GCCAGTTGATTCTTGATCTGGT \\
CCI5 & CGCTTGATCGGCAAAGAAT & CTTCCCGTTGCTTGACGTTG \\
Csf3 & GCTGCTTTGCCTACCTCTCC & TCGAGTGACAAACACGACTGC \\
Mmp9 & GCACTATGGTCAGGACGAGAG & GGGGAAATACCCGATAGAGCC \\
CXC12 & TGTCTGGAGATTCGACTTGAAGTC & TGAGTTCCAGGGCACACCA \\
ZO1 & CCAACCACCAGGCTACAGG 5 GCGTCACACTCAAGCTCTG \\
\hline
\end{tabular}

analyses were performed to explore the significant pathways. All mRNA sequencing data were uploaded to NCBI (accession number PRJNA656088).

\section{Western blot and ELISA}

The tissue lysates were prepared using RIPA lysis buffer, while the cell and exosome lysates were prepared using $3 \times$ Loading Buffer. All samples were performed as depicted previously [6]. Exosome proteins were detected with the antibodies as follows: Alix (1:1000, Cell Signaling Technology, Beverly, MA, USA), Calnexin (1:1000, Cell Signaling Technology, Beverly, MA, USA), CD9 (1: 500, Santa Cruz Biotechnology, Dallas, Texas, USA), ANGPTL1 (1:500, Santa Cruz Biotechnology, Dallas, Texas, USA), TSG101 (1:1000, Abcam, Shanghai, China), ANGPTL1 (1:1000, R\&D Systems, Minneapolis, MN, USA). ImKC proteins were detected with the antibodies as follows: STAT3 (1:1000, Cell Signaling Technology, Beverly, MA, USA), phospho-STAT3 (Y705) (1:1000, Cell Signaling Technology, Beverly, MA, USA), JAK2 (1: 1000, Cell Signaling Technology, Beverly, MA, USA), phospho-JAK2 (Y1008) (1:1000, Cell Signaling Technology, Beverly, MA, USA), GAPDH (1: 2500, Huabio, Hangzhou, China). HUVECs proteins were detected using the antibodies listed as follows: Claudin-5 (1:1000, Abcam, Shanghai, China), ZO-1 (1:1000, Proteintech Group, Rosemont, USA). All samples were further incubated with the peroxidase-conjugated secondary antibody (1:5000, Huabio, Hangzhou, China). Bands were visualized using enhanced chemiluminescence reagents (YEASEN, Shanghai, China) and scanned via a Tanon 5200 Chemiluminescent Imaging System (Tanon, Shanghai, China). Quantitative analysis of WB was performed with ImageJ software (ImageJ v1.8.0).

In order to detect the protein level in the $\mathrm{CM}$ of $\operatorname{ImKC}$ through ELISA, cells were cultured in the presence of exosomes $(10 \mu \mathrm{g} / \mathrm{mL})$ for $24 \mathrm{~h}$ before supernatants were collected, and the MMP9 level was measured by mouse MMP9 ELISA kit (R\&D Systems, Minneapolis, MN, USA).

\section{Immunofluorescence}

For histological analysis, tissues were dissected and fixed in a mix of $2 \%$ Paraformaldehyde (PFA) and 20\% sucrose solution overnight, followed by embedding and section. Then $6 \mu \mathrm{m}$ O.C.T. tissue cryosections were stained with antibodies against F4/80 (1:100, eBioscience, Shanghai, China), $\alpha$ SMA (1:500, Abcam, Shanghai, China), CD31 (1: 200, Abcam, Shanghai, China), MMP9 (1:200, Abcam, Shanghai, China). Secondary antibodies conjugated to Alexa Fluor 488, 555, or 594 were used (1:500, Abcam, Shanghai, China). Fluorescent images were obtained using a Zeiss LSM 710 laser confocal microscope (Carl Zeiss, Germany) and analyzed using Zen software (ZEN 3.0).

For ZO-1 detection in HUVECs, cell immunofluorescence was conducted as described previously [32]. Cells were incubated with primary antibody ZO-1 (1:400, Proteintech Group, Rosemont, USA) at $4{ }^{\circ} \mathrm{C}$ overnight. $\mathrm{Nu}$ clei were stained with DAPI for $5 \mathrm{~min}$. Cells were viewed under the Zeiss LSM 710 laser confocal microscope (Carl Zeiss, Germany) and analyzed using Zen software (ZEN 3.0).

\section{Statistics}

All data are presented as the mean \pm standard error of the mean (SEM) or mean \pm standard deviation of the mean (SD). Statistical analyses were performed with Student's t-test for comparisons between two groups and with ANOVA for more than two groups using Prism 8.0. $P<0.05$ was considered statistically significant.

\section{Results \\ ANGPTL1 level was downregulated in CRC derived exosomes}

To study the ANGPTL1 expression in CRC-derived exosomes, we first detected the ANGPTL1 protein level in exosomes secreted by CRC tumors (TDEs) and paired normal tissues (NDEs). We collected eight pairs of samples from our oncology center and isolated the tissuederived exosomes by ultracentrifugation. The characteristic of TDEs and NDEs were accessed by TEM, DLS, 
and Western Blot (WB) (Fig. 1a-c). We observed a cupshaped structure in both TDEs and NDEs, with the diameter of most particles around $150 \mathrm{~nm}$. WB analysis of the exosomal proteins showed that ANGPTL1 level was significantly decreased in TDEs than in paired NDEs $(P=0.03$, Fig. $1 \mathrm{~d}$ and e, see the detailed ANGPTL1 index of each sample in additional file 4 Table S1). What is more, the ANGPTL1 level in TDEs varied among CRC patients, indicating the patient heterogeneity of exosomal ANGPTL1 levels. The above results suggested that ANGPTL1 was downregulated in CRC derived exosomes, and exosomal ANGPTL1 may be involved in CRC progression.

\section{Exosomal ANGPTL1 attenuated liver metastasis and impeded vascular leakiness in the liver PMN}

In our previous study [6], ANGPTL1 overexpression inhibited the migration and invasion of CRC cells and hindered liver metastasis. To further investigate the function of exosomal ANGPTL1 in CRC, SW620-ANGPTL1 cells were used to enrich the exosomal ANGPTL1. Exosomes were collected from the CM of SW620 (named Ctrl-Exo) and SW620-ANGPTL1 (named ANGPTL1-Exo) through ultracentrifugation (Additional file 1 Fig. S1a and S1b). WB analysis confirmed that ANGPTL1 was abundant in ANGPTL1-Exo (Additional file 1 Fig. S1c).
We then employed an intrasplenic injection mouse model to investigate the role of exosomal ANGPTL1 in liver metastasis. Firstly, SCID-Beige mice were injected retro-orbitally every other day for 21 days with PBS, Ctrl-Exo, or ANGPTL1-Exo in a process that is defined as "education" (Fig. 2a). After that, $2 \times 10^{6}$ SW620 cells were injected into the mouse spleens. The IVIS Lumina imaging system was used to monitor the liver metastasis weekly. At the fourth week, we found the fluorescence intensity in mouse livers was lower in the ANGPTL1Exo education group, compared with the Ctrl-Exo education group $(P=0.02$, Fig. $2 b)$. Then mice were sacrificed, and their livers were harvested for H\&E examination (Fig. 2c). The liver macrometastatic burden was measured by lesion areas. We found a heavier metastatic burden in mouse livers educated by Ctrl-Exo than by PBS or ANGPTL1-Exo (PBS vs Ctrl-Exo, $P<$ 0.01; Ctrl-Exo vs ANGPTL1-Exo, $P<0.01$; Fig. 2c). The results suggested that exosomal ANGPTL1 attenuated liver metastasis induced by tumor-derived exosomes in CRC.

It is known that cancer cells spread through the body in multiple steps [33]. Our intrasplenic mouse model allowed tumor cells to immediately disseminate through the portal circulation after injection [30], making tumor extravasation the first step during liver metastasis. $\mathbf{a}$
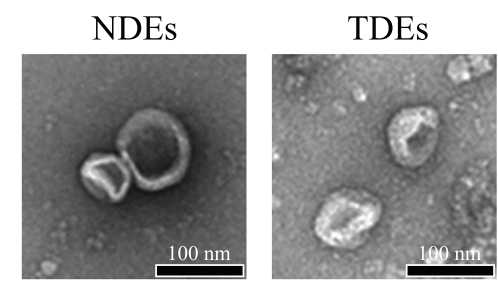

b

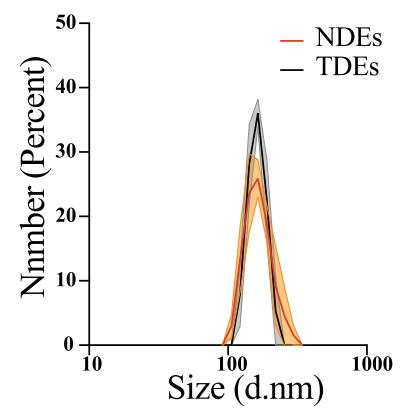

c

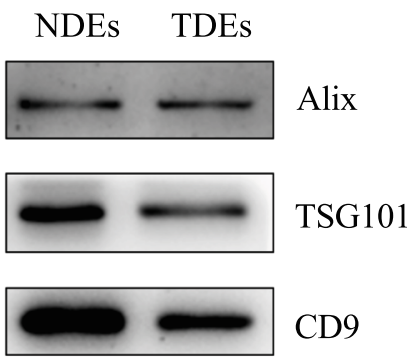

d

$\mathbf{e}$

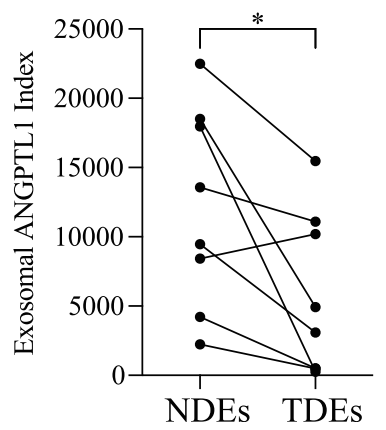

Fig. 1 ANGPTL1 level was downregulated in CRC derived exosomes. a Transmission electron microscopy analysis of TDEs and NDEs. Scale bar, 100 $\mathrm{nm}$. $\mathbf{b}$ The number of particles and median diameter was determined by dynamic light scattering (DLS) analysis. Data were shown as mean \pm standard deviation of three technical replicates. c Western blot (WB) analysis of exosome biomarkers (Alix, TSG101, and CD9). d, e WB analysis of the ANGPTL1 in tissue-derived exosomes, $n=8, P=0.03$. ANGPTL1 level was downregulated in exosomes derived from CRC tumors than paired normal tissues. Data were analyzed using a ratio paired t-test 


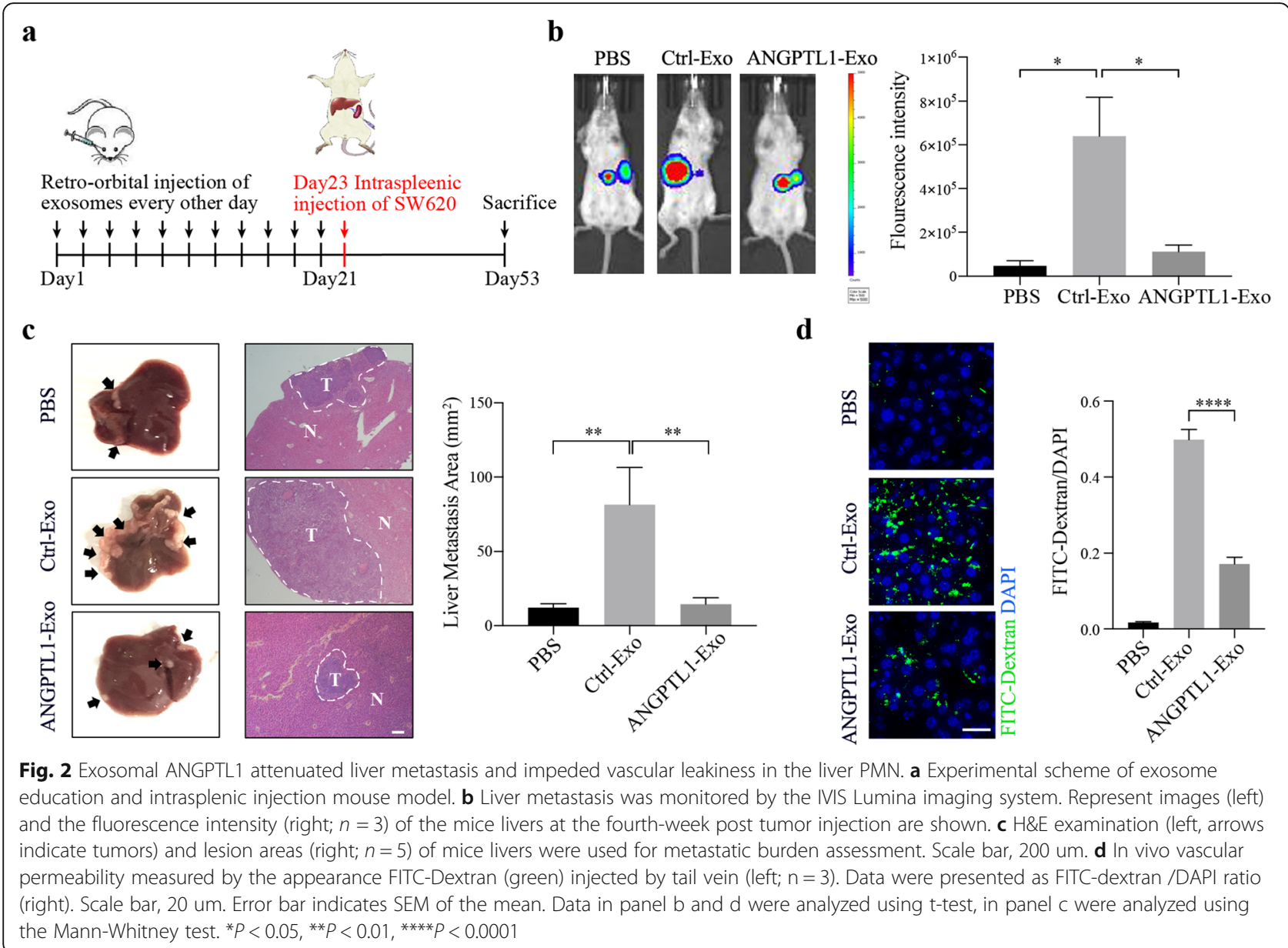

Vascular permeability is one of the most critical factors in this process, and a typical characteristic of PMNs [34]. Furthermore, ANGPTL1 was proved to regulate angiogenesis [7]. Thus, we focused on exosomal ANGP TL1's function on liver vascular permeability. The effect of Ctrl-Exo and ANGPTL1-Exo on endothelial barriers was then detected in vivo. Mice educated by PBS or exosomes were injected with FITC-Dextran through tail veins. More fluorescence was detected in the liver educated by Ctrl-Exo than by PBS. In comparison, less fluorescence was detected in the liver educated by ANGPTL1-Exo $(P<0.001$, Fig. $2 \mathrm{~d})$, which indicated that exosomal ANGPTL1 could impede the vascular leakiness in liver PMNs induced by tumor-derived exosomes.

\section{Exosomal ANGPTL1 was mainly taken up by KCs and regulated $\mathrm{KC}$ s secretion pattern}

To explore the mechanism of how exosomal ANGPTL1 inhibits CRC liver metastasis, we first try to determine the cells that take up CRC derived exosomes in the liver. The PKH67 labeled Ctrl-Exo and ANGPTL1-Exo were injected intravenously. Twenty-four hours post-injection, mouse livers were harvested, and liver frozen sections were analyzed by immunofluorescence. We found the cells which took up exosomes were mainly F4/80 positive, a surface marker of KCs (Fig. 3a). Besides, the labeled exosomes failed to fuse with other cells in the liver microenvironment, such as $\alpha \mathrm{SMA}^{+}$hepatic stellate cells or $\mathrm{CD}_{3} 1^{+}$endothelial cells (Additional file 2 Fig. S2a and $\mathrm{S} 2 \mathrm{~b})$. To validate the $\mathrm{KC}$-specific localization of exosomes, we treated the mice with liposome clodronate, known to deplete macrophages, via tail vein injection. We found that $48 \mathrm{~h}$ of treatment was enough to ablate most of the $\mathrm{F} 4 / 80^{+}$cells in the mouse liver (Additional file 2 Fig. S2c). Then, the PKH67 labeled exosomes were injected into the mice. We found no exosome remained in livers after macrophage ablation (Fig. 3b), indicating that $\mathrm{KC}$ is the predominant cell taking up CRC derived exosomes. Also, we examined the exosome uptake of KCs in vitro. PKH67 labeled Ctrl-Exo and ANGPTL1Exo were co-cultured with $\operatorname{ImKC}$ and were both taken by ImKC in $2 \mathrm{~h}$ (Additional file 2 Fig. S2d) and stably fused into ImKC in $4 \mathrm{~h}$ (Fig. 3c).

The above results implied that exosomal ANGPTL1 might inhibit CRC liver metastasis through regulating KCs. To further investigate the effect of exosomal 


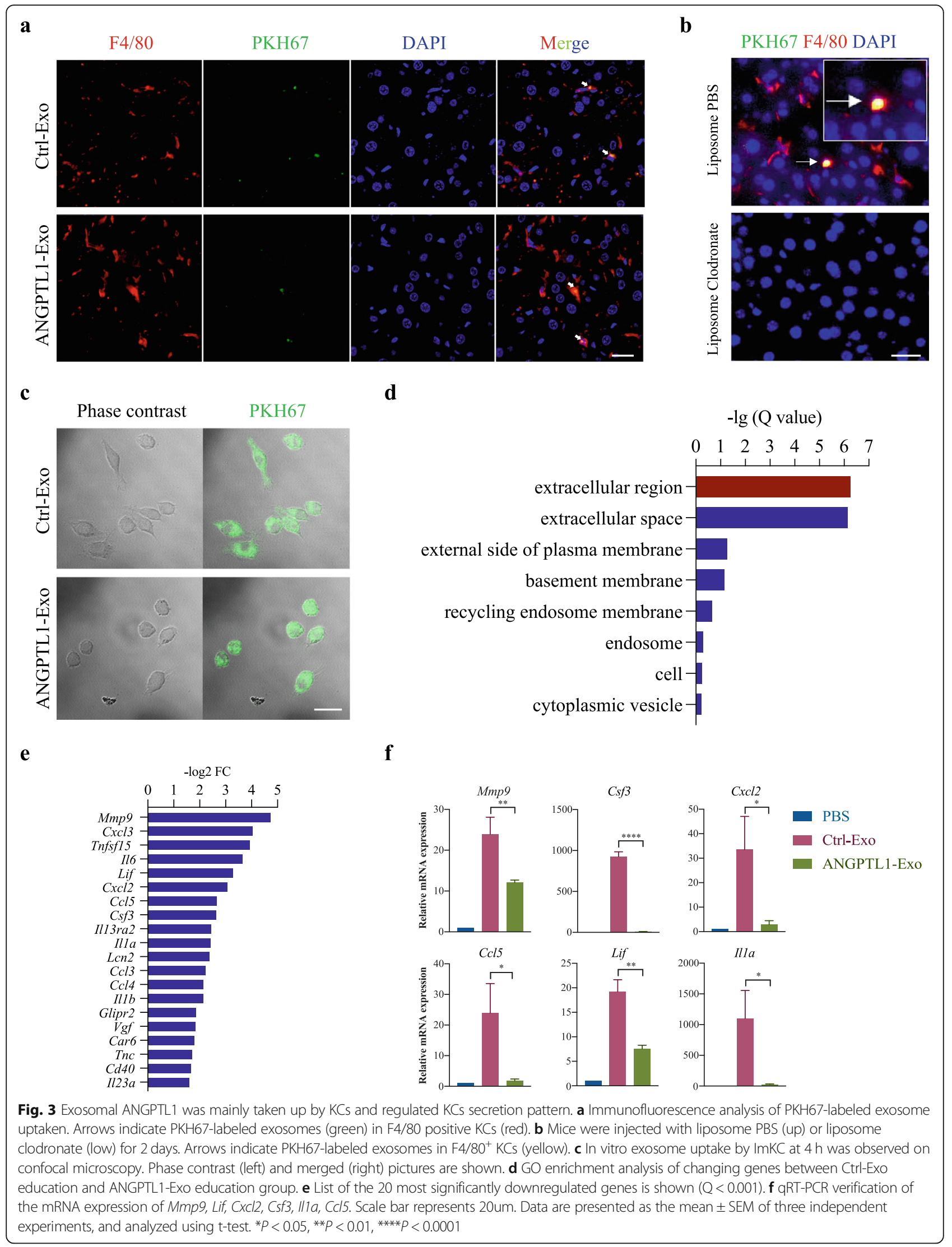


ANGPTL1 on KCs, we educated ImKC with PBS, CtrlExo, and ANGPTL1-Exo in vitro for $24 \mathrm{~h}$ and analyzed gene expression by mRNA sequencing. GO enrichment analysis showed that the changing genes between ImKC educated by Ctrl-Exo and ANGPTL1-Exo mainly belonged to the extracellular region (Fig. 3d), which suggested that the secretion pattern of KCs was regulated. Among the top20 genes (Fig. 3e), Mmp9, Lif, Cxcl2, Csf3, Illa, and Ccl5 were verified by qRT-PCR (Fig. 3f, $P$ $<0.05$ ). The six genes were significantly downregulated in ImKC educated by ANGPTL1-Exo, and all of them were reported to be involved in PMNs formation. Taken together, we found that exosomal ANGPTL1 was mainly taken up by $\mathrm{KCs}$ and regulated KCs secretion pattern, which may result in liver PMNs remodeling.

\section{Exosomal ANGPTL1 dependent MMP9 decrease in KCs normalized vascular leakiness induced by CRC derived exosomes}

Matrix Metallopeptidase 9 (MMP9) is intimately involved in regulating vascular integrity in PMNs [23]. To explore the effect of exosomal ANGPTL1 dependent MMP9 decrease on vascular leakiness, we first verified the MMP9 downregulation in KCs. The ELISA analysis showed that MMP9 was significantly decreased in the CM of ImKC educated by ANGPTL1-Exo than by CtrlExo (Fig. 4a, $P<0.05$ ). We also confirmed that the MMP9 decrease in ANGPTL1-Exo educated mouse livers (Additional file 3 Fig. S3a and S3b). Next, we accessed the permeability of endothelial monolayer after treatment with the $\mathrm{CM}$ of $\mathrm{ImKC}$ by measuring the passage of FITC-Dextran (70 KD). We found that the CM of ANGPTL1-Exo educated ImKC significantly decreased fluorescent probe leakiness as compared to the CM of Ctrl-Exo educated ImKC (Fig. 4b). We then silenced MMP9 expression in ImKC by siRNA transfection $24 \mathrm{~h}$ after Ctrl-Exo education (Additional file 3 Fig. S3c). The CM from MMP9 silencing ImKC caused less fluorescence passage than scramble siRNA (Fig. 4c), which was consistent with the effect of the $\mathrm{CM}$ from ANGPTL1-Exo educated ImKC. Moreover, active rmMMP9 was added into the CM of ImKC educated by ANGPTL1-Exo and resulted in more fluorescent probe passing through the endothelial cell (EC) layer (Fig. 4c). The same tendency was observed in the in vivo vascular permeability assay, and rmMMP9 treatment deteriorated liver vascular leakiness in the mice educated by ANGP TL1-Exo $(P<0.001$, Fig. 4d).

Since the tight junction proteins (TJs) were proved to regulate the EC layer permeability [35], we examined the TJs levels in ECs. The results showed that ZO-1 and Claudin-5 was upregulated in HUVECs treated by the CM of ImKC educated by ANGPTL1-Exo than by CtrlExo. The effect was abolished by extra rmMMP9 (Fig. 4e and $\mathrm{f}$. Immunofluorescence analysis also revealed that rmMMP9 dampened the ZO-1 upregulation in HUVEC monolayers after treatment with CM of ANGPTL1-Exo educated ImKC (Fig. 4g). All the above data above indicated that MMP9 was involved in exosomal ANGPTL1 dependent vascular leakiness prevention.

\section{Exosomal ANGPTL1 downregulated MMP9 in KCs by inhibiting the JAK2-STAT3 signaling pathway}

To further investigate how exosomal ANGPTL1 downregulated MMP9 expression in KCs, we conducted KEGG enrichment to analyze the RNA sequencing data. The results showed that the changing genes between ImKC educated by Ctrl-Exo and ANGPTL1-Exo were mainly enriched in IL-17, TNF, Toll-like receptor, and JAKSTAT signaling pathway (Fig. 5a). Since ANGPTL1 was reported to inhibit the JAK2-STAT3 pathway [18], we focused on the effect of exosomal ANGPTL1 on the JAK2STAT3 pathway. WB analysis showed that ANGPTL1Exo obviously inhibited the phosphorylation of STAT3 (Y705) and JAK2 (Y1008) in KCs at 6, 12, 24h (Fig. 5b and c). MMP9 is known as one of the target genes of STAT3 [36]. To confirm if the JAK2-STAT3 signaling pathway involved in exosomal ANGPTL1 induced MMP9 downregulation in KCs, the recombinant mouse IL-6 was used to active $\operatorname{ImKC}$, which is a specific activator of STAT3. The WB results showed that IL- 6 activated the phosphorylation of STAT3 (Y705) and increased the MMP9 mRNA expression in ImKC educated by ANGT PL1-Exo in 24h (Fig. 5d). The above data demonstrated that exosomal ANGPTL1 downregulated MMP9 in KCs by inhibiting the JAK2-STAT3 pathway, which may be the mechanism of exosomal ANGPTL1 dependent vascular leakiness prevention and liver metastasis attenuation.

\section{Discussion}

ANGPTL1 has been reported to suppress tumor metastasis in several cancers [7], while its extracellular effects on the PMNs are still unclear. Our present study was intended to determine the function of exosomal ANGP TL1 in CRC liver metastasis.

In this study, we found that ANGPTL1 expression was downregulated in the exosomes derived from CRC tumor tissues than paired normal colorectal tissues. The exosomes containing more ANGPTL1 proteins attenuated liver metastasis and impeded vascular leakiness. Further exploration showed that exosomal ANGPTL1 regulated the KCs secretion pattern, significantly decreased the MMP9 expression by inhibiting the JAK2-STAT3 pathway, which in turn normalized vascular leakiness in live PMN (Fig. 5e).

An increasing number of studies have found that ANGPTL1 could be secreted in exosomes. Sinha A et al. identified ANGPTL1 in human ovarian cancer cell- 


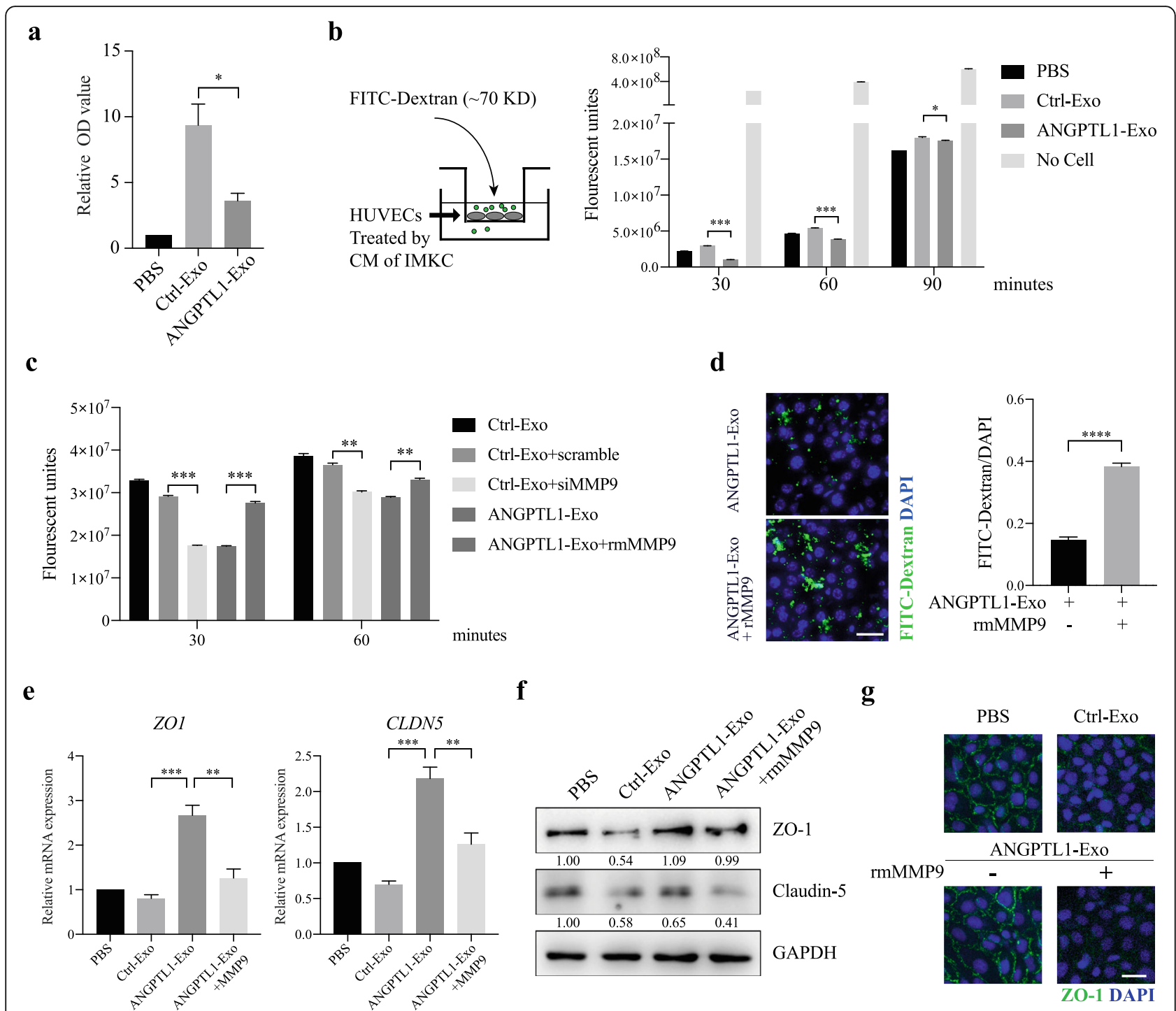

Fig. 4 Exosomal ANGPTL1 dependent MMP9 decrease normalized vascular leakiness induced by CRC derived exosomes. a ELISA analysis of MMP9 level in the culture medium (CM) from ImKC educated by PBS, Ctrl-Exo or ANGPTL1-Exo for $24 \mathrm{~h}$. b, c Permeability of the HUVEC monolayers to FITC-Dextran (70 kDa) after exposure to CM from ImKC educated by PBS, Ctrl-Exo, ANGPTL1-Exo (b), or exposure to CM from ImKC educated by Ctrl-Exo followed by transfection of control siRNA (scramble) or MMP9 siRNA or by ANGPTL1-Exo with extra rmMMP9 (100 ng/mL) (c). The fluorescence in the bottom well was detected every 30 min. $\mathbf{d}$ rmMMP9 $(50 \mu \mathrm{g} / \mathrm{kg})$ was injected through the tail vein into the mice educated by ANGPTL1-Exo for $1 \mathrm{~h}$. In vivo vascular permeability was then determined $(\mathrm{n}=3)$. Representative images are shown (left). Data were presented as FITC-dextran /DAPI ratio (right). e, $\mathbf{f}$ ZO-1 and Claudin-5 expression in HUVECs treated by ImKC CM were detected through GRT-PCR (e) and WB (f). $\mathbf{g}$ Immunofluorescence staining of ZO-1 in HUVECs monolayers treated by ImKC CM. Data are presented as the mean \pm SEM of three independent experiments. Data in panel a, $\mathrm{d}$ and e were analyzed using t-test, in panel $\mathrm{b}$ and $\mathrm{c}$ were analyzed using ANOVA. The scale bar represents 20 um. ${ }^{*} P<0.05,{ }^{* *} P<0.01,{ }^{* * *} P<0.001,{ }^{* * *} P<0.0001$

derived exosomes by mass spectrometry [28]. ANGPTL1 was also detected in exosomes derived from human saliva [26] and urine [27]. However, the role of exosomal ANGPTL1 is unknown. Our study demonstrated for the first time that ANGPTL1 expression was downregulated in exosomes derived from CRC tumors than paired normal tissues. It was similar to its differential expression in CRC tumors and normal tissues [6, 37], suggesting a possible suppression role of exosomal ANGPTL1 on CRC progression.
Our functional experiments in mouse models of CRC proved that the exosomal ANGPTL1 upregulation inhibited liver metastasis. Consistently, early studies have proved that ANGPTL1 hindered tumor metastasis in lung cancer [16], hepatocellular carcinoma (HCC) [18], and CRC [6]. But the mechanism varies. Actually, cancer metastasis is a multi-step process [33]. So far, researchers mostly focus on ANGPTL1's impact on the primary sites, such as tumor invasiveness and mobility inhibition, which prevent tumor cells from invading 
$\mathbf{a}$

$$
\begin{aligned}
& -\lg \text { (Q value) } \\
& \begin{array}{lllll}
0 & 5 & 10 & 15 & 20
\end{array} \\
& \text { Cytokine-cytokine receptor interaction- } \\
& \text { IL-17 signaling pathway- } \\
& \text { TNF signaling pathway }
\end{aligned}
$$

c
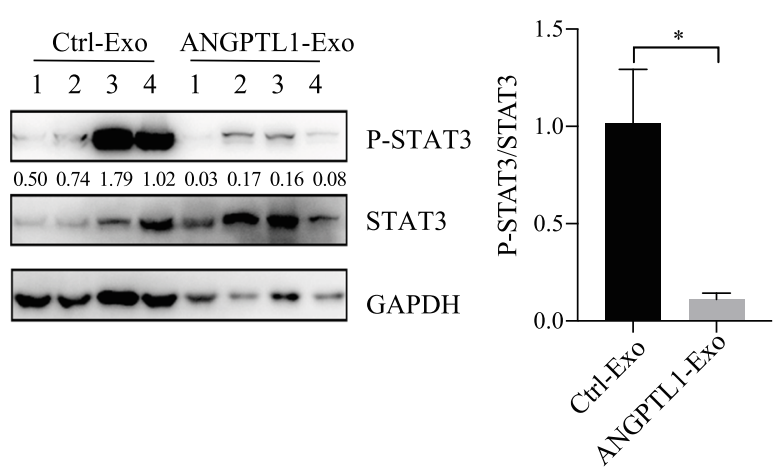

b

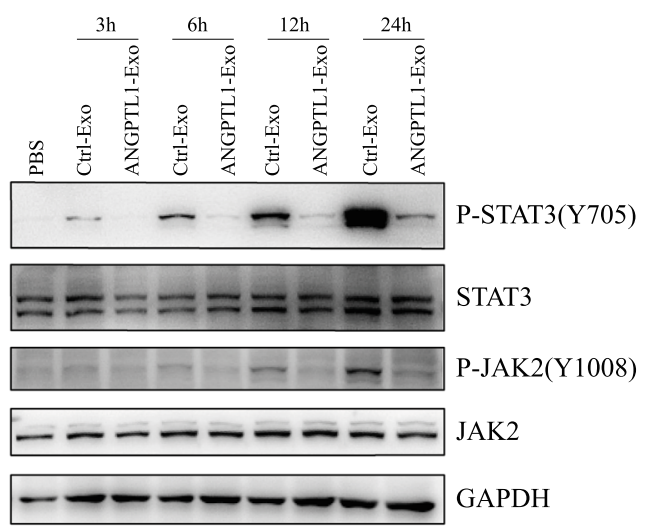

d
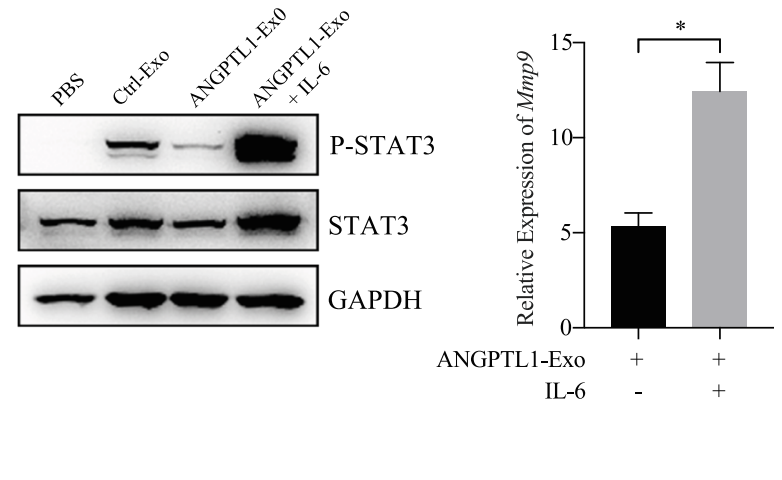

$\mathbf{e}$

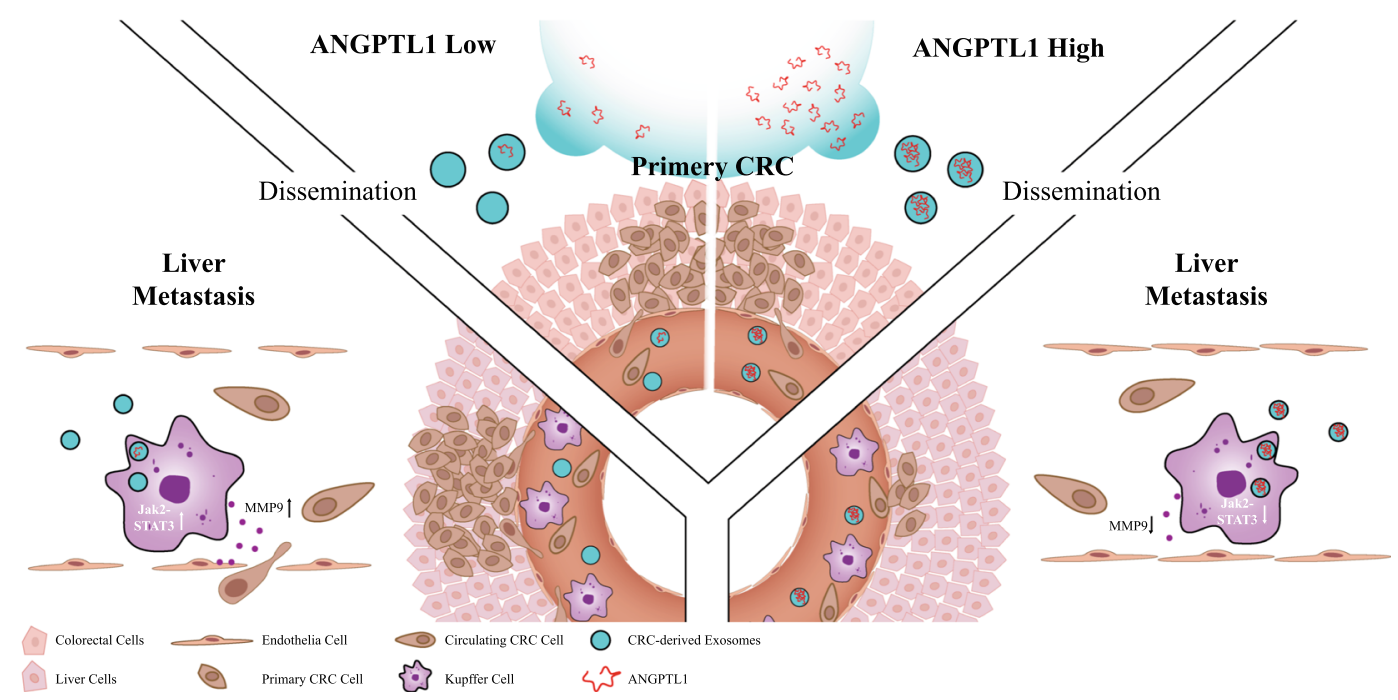

Fig. 5 Exosomal ANGPTL1 decrease MMP9 production in KCs by inhibiting the JAK2-STAT3 signaling pathway. a KEGG pathway enrichment analysis of the changing genes between ImKC educated by Ctrl-EXo and ANGPTL1-EXO. The top 11 significant pathways were shown. $\mathbf{b}$ WB validation of the JAK2-STAT3 pathway in IMK educated by PBS, Ctrl-ExO and ANGPTL1-EXO at different times. $\mathbf{c}$ WB analysis of STAT3 phosphorylation in liver tissues from mice educated by PBS, Ctrl-Exo and ANGPTL1-EXo for 3 weeks (right). The number under the first band indicates the value of P-STAT3/STAT3 and the data were analyzed using t-test (left). $\mathbf{d}$ WB validation of STAT3 activation induced by IL-6 (left) and qRT-PCR analysis of MMP9 expression (right) in ImKC treated by ANGPTL1-EXo with or without IL-6 for $24 \mathrm{~h}$. e Schematic diagram of the role of exosomal ANGPTL1 in CRC liver metastasis. Data are presented as the mean \pm SEM of three independent experiments, and analyzed using t-test. ${ }^{*} P<0.05$ 
nearby tissues and moving through the vascular walls $[15,16,18]$. However, we paid our attention to the metastatic organs and found exosomal ANGPTL1 impeded liver vascular leakiness induced by CRC derived exosomes. Growing evidence indicates that exosomes serve as mediators for long-distance cell-to-cell communication and play a pivotal part in PMNs formation [20]. Several studies have shown increased vascular permeability at PMNs, including liver PMN, which is associated with an enhanced metastatic burden [34]. For example, the melanoma-derived exosomes increased the metastatic behavior of primary tumors by inducing vascular leakiness at pre-metastatic sites [38]; breast cancer-derived exosomes destroyed vascular endothelial barriers to promote metastasis [39]. Thus, it may be reasonable to imply that exosomal ANGP TL1 attenuated CRC liver metastasis by preventing liver vascular leakiness. However, whether exosomal ANGPTL1 affected vascular permeability through a direct effect on the endothelial cells or other indirect way is still unknown.

Our study found that $\mathrm{KC}$ was the predominant cell that took up CRC-derived exosomes in the liver, which was also approved by Shao Yingkuan et al. [40]. It indicated that exosomal ANGPTL1 possibly remodeled the liver PMNs through KCs. Several studies have correlated KCs with PMNs formation and liver metastasis [41, 42]. Once reprogrammed by tumor-derived exosomes, KCs exert their regurgitation-feeding activity on liver microenvironments via the secretion of cytokines and chemokines $[40,43]$. We detected an evident variation of the secretion profile of factors from KCs upon exposure to exosomal ANGPTL1, which influenced the liver PMN formation. Among these changing factors, we attributed MMP9 to exosomal ANGPTL1 dependent vascular leakiness prevention. The ANGPTLs were reported to regulate the expression of MMP9 in osteosarcoma [44] and HCC [45]. Moreover, MMP9 is known to be involved in regulating vascular integrity in PMNs [23]. High levels of MMP9 in the premetastatic lung promoted vascular remodeling, while genetic ablation of MMP9 normalized the aberrant vasculature in the lung PMN, impeding cancer metastasis [46]. The above evidence suggested that exosomal ANGPTL1 reprogrammed $\mathrm{KC}$ and downregulated its MMP9 expression, thus preventing liver vascular leakiness and hindering CRC liver metastasis.

Like proteins or microRNAs, exosomal cargos can regulate the recipient cells' physiological activities [47]. We demonstrated that exosomal ANGPTL1 inhibited the JAK2-STAT3 signal pathway, especially hindered STAT3 activation. The exosomal ANGPTL1 dependent MMP9 downregulation was reversed by IL6 induced STAT3 activation. It indicated that exosomal ANGPTL1 downregulated the MMP9 expression by inhibiting the JAK2-STAT3 signaling pathway. Consistently, Qian Yan et al. also found ANGPTL1 repressed the JAK-STAT3 signaling in HCC [18]. There is substantial evidence confirming that the JAK-STAT3 signaling pathway is involved in CRC development [48]. STAT3 (Signal transducer and activator of transcription 3) is a critical transcriptional factor identified as a central regulator of tumor metastasis. STAT3 could be activated by IL-6 and promote gene transcription, including MMP9 [36]. Therefore, we implied that exosomal ANGPTL1 might influence liver vascular permeability through the JAK2-STAT3-MMP9 axis.

However, how exosomal ANGPTL1 inhibited the JAK2-STAT3 pathway still needs further investigation. The way that exosomes transfer the content or induce signals may involve ligand-receptor interaction or cytomembrane fusion [20]. Several studies have shown that ANGPTL proteins deliver their signals via integrin receptor-related pathways $[16,18]$. The previous study proved that ANGPTL1 interacted with the integrin $\alpha 1 \beta 1$ receptor to suppress the downstream FAK/Src-JAK-STAT3 signaling pathway [18]. Besides, ANGPTL1 worked intracellular to represses the SrcJAK-STAT3 signaling [18]. Therefore, we implied that the possible mechanism of exosomal ANGPTL1 inhibiting JAK2-STAT3 pathway might be: (1) ANGPTL1 exists on the exosome membrane and binds the integrin receptors on the KCs membrane, suppressing the downstream JAK2-STAT3 signaling pathway; (2) exosomes fuse with the KCs membrane, and ANGPTL1 is released into the cytoplasm, inhibiting the JAK2STAT3 pathway through interaction with molecules involved in the pathway. These questions will be explored in our future research.

\section{Conclusion}

This study aimed to explore the function of exosomal ANGPTL1 in CRC liver metastasis. Our experimental results demonstrated that ANGPTL1 was downregulated in CRC derived exosomes. More importantly, exosomal ANGPTL1 attenuated CRC liver metastasis and impeded vascular leakiness. In mechanism, we found exosomal reprogrammed the Kupffer cell and decreased MMP9 expression through inhibiting the JAK2-STAT3 signaling pathway. These findings suggest a suppression role of exosomal ANGPTL1 on CRC progression, and expand the approach of ANGP TL1 functioning, enriching the mechanisms of CRC liver metastasis. 


\section{Supplementary Information}

The online version contains supplementary material available at https://doi. org/10.1186/s13046-020-01816-3.

Additional file 1: Fig. S1. Characterization of exosomes derived from SW620-Ctrl and SW620-ANGPTL1 cells. a Electron microscopy analysis of Ctrl-Exo and ANGPTL1-Exo. Scale bar, $100 \mathrm{~nm}$. b The number of particles and median diameter was determined by dynamic light scattering (DLS) analysis. $n=3$. Data were shown as mean \pm standard deviation of three technical replicates. c Western blot analysis of exosomes positive biomarkers (Alix, TSG101), negative biomarkers (Calnexin), and exosomal ANGPTL1.

Additional file 2: Fig. S2. Immunofluorescent validation of the KCspecific localization of exosomes. $a$, b Immunofluorescence analysis of the colocalization of PKH67-labelled exosome (green) with aSMA ${ }^{+}$Hepatic stellate cells (hStCs) (A) or CD31 ${ }^{+}$endothelial cells (ECs) (B) (red). Arrows indicate PKH67-labelled exosomes without fusion with hStCs or ECs. c Mice were injected with PBS (up) or liposome clodronate (low) for 2 days. Immunostaining of F4/80+ $\mathrm{KCs}$ (red) showed complete ablation of KCs in mouse liver after treated with liposome clodronate. $d$ In vitro exosome uptake by ImKC at $2 \mathrm{~h}$ was observed on confocal microscopy. Phase contrast (left) and merged (right) pictures are shown

Additional file 3: Fig. S3. $q R T-P C R$, immunofluorescent, ELISA validation of MMP9 expression. a qRT-PCR validation of MMP9 expression in liver tissue from the mice educated by PBS, Ctrl-Exo or ANGPTL1-EXo, $n=3$. b Immunofluorescence analysis of MMP9 in liver slices from the mice education by PBS, Ctrl-EXo or ANGPTL1-EXo, $n=3$. c qRT-PCR (left) and ELISA (right) validation of MMP9 silencing in ImKC. Data are presented as the mean \pm SD of three independent experiment, and analyzed using t-test. * $P<0.05$.

Additional file 4: Table S1. The stages of CRC patients and the corresponding exosomal ANGPTL1 index calculated by ImageJ from WB brands. The CRC stage was based on 7th revised edition of the AJCC Colorectal Cancer.

\section{Abbreviations}

CRC: colorectal cancer; CLM: colorectal liver metastasis; PMNs: pre-metastatic niches; PBS: Phosphate buffer saline; OD: Optical density; ELISA: Enzymelinked immunosorbent assay; SD: Standard deviation; SEM: standard error of the mean; ANGPTL1: Angiopoietin-like protein 1; HCC: Hepatocellular Carcinoma; PDAC: Pancreatic ductal adenocarcinoma; KCs: Kupffer cells; HUVEC: Human umbilical vein endothelial cells; ImKC: Immortalized Mouse Kupffer Cells; TDEs: tumor-derived exosomes; NDEs: normal tissue-derived exosomes; TEM: Transmission electron microscopy; DLS: dynamic light scattering; rmMMP9: recombinant mouse MMP9; CM: culture medium; AMPA: aminophenylmercuricacetate; EC: endothelial cell; TJs: tight junction proteins; KEGG: Kyoto Encyclopedia of Genes and Genomes; GO: Gene Ontology; H\&E staining: Hematoxylin and eosin staining

\section{Acknowledgments}

Not applicable.

\section{Authors' contributions}

Kai Jiang, Haiyan Chen, and Yimin Fang designed and conducted most of the experiments in this study and analyzed the data. They contributed equally to this work. Liubo Chen, Chenhan Zhong, Tongtong Bu, Siqi Dai, Xiang Pan, Dongliang Fu, Yucheng Qian, and Jingsun Wei finished the rest part of the experiments in this study. Kefeng Ding provided guidance for this work and revised the manuscript. All authors read and approved the final manuscript.

\section{Funding}

This study was supported by the National Natural Science Foundation of China (Grant No. 81772545, 11932017); the National Key R\&D Program of China (No. 2017YFC0908200); the Key Technology Research and Development Program of Zhejiang Province (No. 2017C03017).

\section{Availability of data and materials}

All data generated or analyzed during this study are included in this published article and its supplementary information files.

\section{Ethics approval and consent to participate}

All experiments containing patient specimens or data were approved by the Ethical Committee of the Second Affiliated Hospital of Zhejiang University School of Medicine and informed consent was obtained from all patients. All animal experiments were approved by the Institutional Ethics Committee of the Second Affiliated Hospital Zhejiang University School of Medicine (Zhejiang, P.R. China).

\section{Consent for publication}

Not applicable.

\section{Competing interests}

The authors declare no competing interest.

\section{Author details}

${ }^{1}$ Department of Colorectal Surgery and Oncology, Key Laboratory of Cancer Prevention and Intervention, Ministry of Education, the Second Affiliated Hospital, Zhejiang University School of Medicine, Hangzhou, Zhejiang, China. ${ }^{2}$ Cancer Institute, Key Laboratory of Cancer Prevention and Intervention, Ministry of Education, The Second Affiliated Hospital, Zhejiang University School of Medicine, Hangzhou, Zhejiang, China. ${ }^{3}$ Department of Medical Oncology, Key Laboratory of Cancer Prevention and Intervention, Ministry of Education, The Second Affiliated Hospital, Zhejiang University School of Medicine, Hangzhou, Zhejiang, China.

Received: 25 August 2020 Accepted: 13 December 2020

Published online: 07 January 2021

\section{References}

1. Torre LA, Bray F, Siegel RL, Ferlay J, Lortet-Tieulent J, Jemal A. Global cancer statistics, 2012. CA Cancer J Clin. 2015;65:87-108.

2. Siegel RL, Miller KD, Jemal A. Cancer statistics, 2015. CA Cancer J Clin. 2015; 65:5-29.

3. Siegel RL, Miller KD, Fedewa SA, Ahnen DJ, Meester RGS, Barzi A, Jemal A. Colorectal cancer statistics, 2017. CA Cancer J Clin. 2017;67:177-93.

4. Miller KD, Siegel RL, Lin CC, Mariotto AB, Kramer JL, Rowland JH, Stein KD, Alteri R, Jemal A. Cancer treatment and survivorship statistics, 2016. CA Cancer J Clin. 2016;66:271-89.

5. Capdevila J, Saura C, Macarulla T, Casado E, Ramos FJ, Tabernero J. Monoclonal antibodies in the treatment of advanced colorectal cancer. Eur J Surg Oncol. 2007:33(Suppl 2):S24-34.

6. Chen H, Xiao Q, Hu Y, Chen L, Jiang K, Tang Y, Tan Y, Hu W, Wang Z, He J, et al. ANGPTL1 attenuates colorectal cancer metastasis by up-regulating microRNA-138. J Exp Clin Cancer Res. 2017:36:78.

7. Carbone C, Piro G, Merz V, Simionato F, Santoro R, Zecchetto C, Tortora G, Melisi D. Angiopoietin-like proteins in angiogenesis, inflammation and Cancer. Int J Mol Sci. 2018;19(2):431.

8. Tabata M, Kadomatsu T, Fukuhara S, Miyata K, Ito Y, Endo M, Urano T, Zhu HJ, Tsukano H, Tazume H, et al. Angiopoietin-like protein 2 promotes chronic adipose tissue inflammation and obesity-related systemic insulin resistance. Cell Metab. 2009:10:178-88.

9. Kersten S. Regulation of lipid metabolism via angiopoietin-like proteins. Biochem Soc Trans. 2005;33:1059-62.

10. Zhang CC, Kaba M, Ge G, Xie K, Tong W, Hug C, Lodish HF. Angiopoietinlike proteins stimulate ex vivo expansion of hematopoietic stem cells. Nat Med. 2006;12:240-5.

11. Sato R, Yamasaki M, Hirai K, Matsubara T, Nomura T, Sato F, Mimata H. Angiopoietin-like protein 2 induces androgen-independent and malignant behavior in human prostate cancer cells. Oncol Rep. 2015;33:58-66.

12. Lim SY, Gordon-Weeks A, Allen D, Kersemans V, Beech J, Smart S, Muschel RJ. Cd11b(+) myeloid cells support hepatic metastasis through downregulation of angiopoietin-like 7 in cancer cells. Hepatology. 2015;62:521-33.

13. Kim I, Kwak HJ, Ahn JE, So JN, Liu M, Koh KN, Koh GY. Molecular cloning and characterization of a novel angiopoietin family protein, angiopoietin-3. FEBS Lett. 1999;443:353-6.

14. Dhanabal M, LaRochelle WJ, Jeffers M, Herrmann J, Rastelli L, McDonald WF, Chillakuru RA, Yang M, Boldog FL, Padigaru M, et al. Angioarrestin: an 
antiangiogenic protein with tumor-inhibiting properties. Cancer Res. 2002; 62:3834-41.

15. Xu Y, Liu YJ, Yu Q. Angiopoietin-3 inhibits pulmonary metastasis by inhibiting tumor angiogenesis. Cancer Res. 2004;64:6119-26.

16. Kuo TC, Tan CT, Chang YW, Hong CC, Lee WJ, Chen MW, Jeng YM, Chiou J, Yu P, Chen PS, et al. Angiopoietin-like protein 1 suppresses SLUG to inhibit cancer cell motility. J Clin Invest. 2013;123:1082-95.

17. Chen HA, Kuo TC, Tseng CF, Ma JT, Yang ST, Yen CJ, Yang CY, Sung SY, Su JL. Angiopoietin-like protein 1 antagonizes MET receptor activity to repress sorafenib resistance and cancer stemness in hepatocellular carcinoma. Hepatology. 2016;64:1637-51.

18. Yan Q, Jiang L, Liu M, Yu D, Zhang Y, Li Y, Fang S, Li Y, Zhu YH, Yuan YF, Guan XY. ANGPTL1 interacts with integrin alpha1beta1 to suppress HCC angiogenesis and metastasis by inhibiting JAK2/STAT3 signaling. Cancer Res. 2017;77:5831-45.

19. Peinado H, Zhang H, Matei IR, Costa-Silva B, Hoshino A, Rodrigues G, Psaila B, Kaplan RN, Bromberg JF, Kang Y, et al. Pre-metastatic niches: organspecific homes for metastases. Nat Rev Cancer. 2017;17:302-17.

20. Kalluri R, LeBleu VS. The biology, function, and biomedical applications of exosomes. Science. 2020;367.

21. Minciacchi VR, Freeman MR, Di Vizio D. Extracellular vesicles in cancer: exosomes, microvesicles and the emerging role of large oncosomes. Semin Cell Dev Biol. 2015:40:41-51.

22. Xie F, Zhou X, Fang M, Li H, Su P, Tu Y, Zhang L, Zhou F. Extracellular vesicles in Cancer immune microenvironment and Cancer immunotherapy. Adv Sci (Weinh). 2019;6:1901779.

23. Kaplan RN, Riba RD, Zacharoulis S, Bramley AH, Vincent L, Costa C, MacDonald DD, Jin DK, Shido K, Kerns SA, et al. VEGFR1-positive haematopoietic bone marrow progenitors initiate the pre-metastatic niche. Nature. 2005;438:820-7.

24. Zeng Z, Li Y, Pan Y, Lan X, Song F, Sun J, Zhou K, Liu X, Ren X, Wang F, et al. Cancer-derived exosomal miR-25-3p promotes pre-metastatic niche formation by inducing vascular permeability and angiogenesis. Nat Commun. 2018;9:5395.

25. Zhou W, Fong MY, Min Y, Somlo G, Liu L, Palomares MR, Yu Y, Chow A, O'Connor ST, Chin AR, et al. Cancer-secreted miR-105 destroys vascular endothelial barriers to promote metastasis. Cancer Cell. 2014;25:501-15.

26. Gonzalez-Begne M, Lu B, Han X, Hagen FK, Hand AR, Melvin JE, Yates JR. Proteomic analysis of human parotid gland Exosomes by multidimensional protein identification technology (MudPIT). J Proteome Res. 2009;8:1304-14.

27. Wang Z, Hill S, Luther JM, Hachey DL, Schey KL. Proteomic analysis of urine exosomes by multidimensional protein identification technology (MudPIT). Proteomics. 2012;12:329-38.

28. Sinha A, Ignatchenko V, Ignatchenko A, Mejia-Guerrero S, Kislinger T. Indepth proteomic analyses of ovarian cancer cell line exosomes reveals differential enrichment of functional categories compared to the NCl 60 proteome. Biochem Biophys Res Commun. 2014;445:694-701.

29. Jiang K, Jiao Y, Liu Y, Fu D, Geng H, Chen L, Chen H, Shen X, Sun L, Ding K. HNF6 promotes tumor growth in colorectal cancer and enhances liver metastasis in mouse model. J Cell Physiol. 2019;234:3675-84.

30. Kozlowski JM, Fidler IJ, Campbell D, Xu ZL, Kaighn ME, Hart IR. Metastatic behavior of human tumor cell lines grown in the nude mouse. Cancer Res. 1984:44:3522-9.

31. Zeisberger SM, Odermatt B, Marty C, Zehnder-Fjallman AH, Ballmer-Hofer K, Schwendener RA. Clodronate-liposome-mediated depletion of tumourassociated macrophages: a new and highly effective antiangiogenic therapy approach. Br J Cancer. 2006;95:272-81.

32. Li J, Li Z, Jiang P, Peng M, Zhang X, Chen K, Liu H, Bi H, Liu X, Li X. Circular RNA IARS (circ-IARS) secreted by pancreatic cancer cells and located within exosomes regulates endothelial monolayer permeability to promote tumor metastasis. J Exp Clin Cancer Res. 2018;37:177.

33. Valastyan S, Weinberg RA. Tumor metastasis: molecular insights and evolving paradigms. Cell. 2011;147:275-92.

34. Huang Y, Song N, Ding Y, Yuan S, Li X, Cai H, Shi H, Luo Y. Pulmonary vascular destabilization in the premetastatic phase facilitates lung metastasis. Cancer Res. 2009;69:7529-37.

35. Ma J, Wang P, Liu Y, Zhao L, Li Z, Xue Y. Kruppel-like factor 4 regulates blood-tumor barrier permeability via ZO-1, occludin and claudin-5. J Cell Physiol. 2014;229:916-26.

36. Dechow TN, Pedranzini L, Leitch A, Leslie K, Gerald WL, Linkov I, Bromberg $J$ J. Requirement of matrix metalloproteinase-9 for the transformation of human mammary epithelial cells by Stat3-C. Proc Natl Acad Sci U S A. 2004; 101:10602-7.

37. Fan H, Huang L, Zhuang X, Ai F, Sun W. Angiopoietin-like protein 1 inhibits epithelial to mesenchymal transition in colorectal cancer cells via suppress Slug expression. Cytotechnology. 2019;71:35-44.

38. Peinado H, Aleckovic M, Lavotshkin S, Matei I, Costa-Silva B, Moreno-Bueno G, Hergueta-Redondo M, Williams C, Garcia-Santos G, Ghajar C, et al. Melanoma exosomes educate bone marrow progenitor cells toward a prometastatic phenotype through MET. Nat Med. 2012;18:883-91.

39. Wang S, Song R, Wang Z, Jing Z, Wang S, Ma J. S100A8/A9 in inflammation. Front Immunol. 2018;9:1298.

40. Shao Y, Chen T, Zheng X, Yang S, Xu K, Chen X, Xu F, Wang L, Shen Y, Wang $T$, et al. Colorectal cancer-derived small extracellular vesicles establish an inflammatory premetastatic niche in liver metastasis. Carcinogenesis. 2018:39:1368-79.

41. Bayon LG, Izquierdo MA, Sirovich I, van Rooijen N, Beelen RH, Meijer S. Role of Kupffer cells in arresting circulating tumor cells and controlling metastatic growth in the liver. Hepatology. 1996;23:1224-31.

42. Wen SW, Ager El, Christophi C. Bimodal role of Kupffer cells during colorectal cancer liver metastasis. Cancer Biol Ther. 2013;14:606-13.

43. Costa-Silva B, Aiello NM, Ocean AJ, Singh S, Zhang H, Thakur BK, Becker A, Hoshino A, Mark MT, Molina H, et al. Pancreatic cancer exosomes initiate pre-metastatic niche formation in the liver. Nat Cell Biol. 2015;17:816-26.

44. Odagiri H, Kadomatsu T, Endo M, Masuda T, Morioka MS, Fukuhara S, Miyamoto T, Kobayashi E, Miyata K, Aoi J, et al. The secreted protein ANGPTL2 promotes metastasis of osteosarcoma cells through integrin alpha5beta1, p38 MAPK, and matrix metalloproteinases. Sci Signal. 2014;7:ra7.

45. Yu H, Zhang H, Li D, Xue H, Pan C, Zhao S, Wang L. Effects of ANGPTL3 antisense oligodeoxynucleotides transfection on the cell growths and invasion of human hepatocellular carcinoma cells. Hepatogastroenterology. 2011;58:1742-6.

46. Yan HH, Pickup M, Pang Y, Gorska AE, Li Z, Chytil A, Geng Y, Gray JW, Moses $\mathrm{HL}$, Yang L. Gr-1+CD11b+ myeloid cells tip the balance of immune protection to tumor promotion in the premetastatic lung. Cancer Res. 2010; 70:6139-49.

47. Mathieu M, Martin-Jaular L, Lavieu G, Thery C. Specificities of secretion and uptake of exosomes and other extracellular vesicles for cell-to-cell communication. Nat Cell Biol. 2019;21:9-17.

48. Yu H, Pardoll D, Jove R. STATs in cancer inflammation and immunity: a leading role for STAT3. Nat Rev Cancer. 2009;9:798-809.

\section{Publisher's Note}

Springer Nature remains neutral with regard to jurisdictional claims in published maps and institutional affiliations.

\section{Ready to submit your research? Choose BMC and benefit from:}

- fast, convenient online submission

- thorough peer review by experienced researchers in your field

- rapid publication on acceptance

- support for research data, including large and complex data types

- gold Open Access which fosters wider collaboration and increased citations

- maximum visibility for your research: over $100 \mathrm{M}$ website views per year

At BMC, research is always in progress.

Learn more biomedcentral.com/submissions 